more or less large extent, which is nothing else than the tracheal sound transmitted by the enlarged glands. It may be heard behind, near the spinal column ; in front, near the sternum ; with this peculiarity, that sometimes the movement of the body may modify this sound, by changing the relation of the trachea with the surrounding parts. I observed this alteration of the tracheal souffle in a remarkable degree, lately, in a female patient in my wards at the Hôtel Dieu. The souffle was very strong when she lifted her head up, and the trachea was closely applied against the convex surface of the cervical spine; it disappeared when she bent her head forward, and the connection between the trachea and the enlarged glands, which conducted the sound, was relaxed. For many weeks I could, according to the position of the neck, produce the souffle, or, on the contrary, make it disappear. The cough, usually the consequence of the bronchial adenopathy, is dry, obstinate, sometimes attended with a long whistling inspiration, as in hooping cough. This hooping cough-like character has been pointed out as belonging to the most advanced degree of this disease by all the physicians who have written on this affection. It may be observed in its mildest forms, and depends very probably on the connection of the enlarged glands with the pneumogastric nerve. I dare not assert that this peculiar character in hooping cough is always the result of the enlargement of these glands; but I found all the signs proper to this condition in some cases of hooping cough, namely, in those which may be called chronic ; and this complication seems to me to explain satisfactorily the unusual circumstance of an indefinite duration in a contagious disease like hooping cough, assimilable in many respects to the eruptive fevers. Everybody knows that the cough may retain its character sometimes for several years ; and, in a case of this description, lasting for two years, I observed the physical signs of the bronchial adenopathy. I have noticed that the cough may disappear in some chronic cases, although all the signs of the bronchial adenopathy have persisted.

The next symptom to be observed is a slight degree of dyspnœa, sometimes recurrent, and assuming an asthmatic form, or only perceptible when the patient is making an exertion, or when some accidental congestion increases the swelling of the glands. I have seen, under such circumstances, a depression of the skin above the superior extremity of the sternum, at each inspiration, as in cases where some obstacle prevents the free access of air to the lungs. The dilatation of the corresponding side of the chest is also diminished, as can be ascertained by a little instrument which I have called a pneuometer, which serves to measure the relative degree of dilatation of both sides of the chest. This instiument is composed of a spring supporting a dial with a graduated index. On each side of the dial is a string, which serves both to fix the instrument in the required position, and also to measure the breadth of the chest.

This morbid condition of the glands explains certain cases of aphonia. I have, on two occasions in my practice, met with cases of aphonia accompanied by all the physical signs of bronchial adenopathy on one side; and from these circumstances I diagnosed a paralysis of the vocal chord of the corresponding side, due to a compression of the inferior laryngeal nerve ; which diagnosis was afterwards verified by a laryngoscopic examination.

In one case, the bronchial adenopathy seemed to me accountable, in a young boy, for an unrestrainable vomiting, attended with dyspnœa, which lasted for two years. Many treatments had been unsuccessfully employed to master this affection. I prescribed Labourboule waters half a glass four times a day, a plaister with belladonna to be applied on the pit of the stomach, and tincture of iodine on the chest; and under this treatment, directed principally against the enlargement of the bronchial glands, the patient was quite cured.

I do not wish to describe the symptoms of the most advanced degrees of this affection, which are well known, when large tumours press on the veins, the trachea, the œesophagus, and produce cynanosis, œdema of the superior parts, congestion of the brain, dysphagia, and sometimes asphyxia.

These periods of this affection which I have studied here are more amenable to treatment than those of more advanced stages. The mineral waters, containing chloride of sodium and arseniate of soda, such as those of Labourboule, or chloride of sodium and iodine, as Kreutsnach, Salies, and Salins, and, in certain cases, sulphurous springs, may be efficaciously prescribed. The use of iodine internally and externally is also indicated.

This condition of the lymphatic glands, to which I have alluded, may be observed at every age ; it may complicate a great number of affections, such as measles, hooping cough, typhoid fever, bronchitis, pleurisy, pneumonia, tubercular induration of the lungs, and accounts for many auscultatory phenomena which I had been previously at a loss to explain.
ON THE NATURE OF THE SO-CALLED BRONCHIAL ASTHMA.*

By J. B. BERKART, M.D.,

Assistant-Physician to the Victoria Park Hospital for Diseases of the Chest.

Tire history of the so-called bronchial asthma dates from the time of Laennec, and with it also the uncertainty with regard to its nature. Whilst up to this period almost every dyspnœa was designated as asthma, Laennec recognised the true value of the various species of it which were in vogue at his time, and relegated them to their proper places as symptoms of anatomically defined diseases. He was enabled to do so because he appreciated the objective signs, which indicate rather "coarse" changes in the physical constitution of the organs of respiration and circulation, and inferred from them the anatomical conditions which produce the dyspnœa and the other symptoms accompanying this.

This means, however, which served to recognise the pathological state in all cases of dyspncea, failed even in the hands of Laennec with regard to one, which occurs in paroxysms, and is followed by intervals of a respiration completely healthy, or almost so. Although that dys. pnœa most frequently occurs in connection with bronchitis and emphysema, and although these are able to sufficiently account for dyspncea in general, yet the origin of the paroxysm and the cause of the free interval remained a perfect mystery. Moreover, cases came under Laennec's notice where even post mortem examination gave negative results. The cases referred to, which have noihing in common with asthma but the sudden dyspnœa, would, we may confidently say, be interpreted to-day in a different way to what they were at that time ; and Laennec could only escape the difficulties which beset him by having recourse to the nervous system, and by attributing the paroxysmal dyspnœa to a dis. turbance of innervation.

Laennec's views on the subject have been adopted by the majority of his successors. Often have weighty objections been raised to the neurotic nature of asthma, but always has the nervous system triumphantly shielded its devout followers, until at last the asthma assumed the formidable character of a spasmodic disease, a theory which, at the present day, counts the largest number of adherents.

Such success, however, was only possible because, on the one hand, the absence of physical signs was supposed to prove the healthy conditions of the lungs beyond a doubt ; and, on the other hand, because experimental physiology appeared to have provided the spasm-theory with the stamp of genuineness.

But neither of these circumstances deserves at our hands the value which has erroneously been attributed to them. For the absence of physical signs is no safe indicator of the absence also of pathological conditions, and all the positive evidence derived from experimental physiology only goes to show that the "bronchial muscles may contract"; but as to "how" and as to "when" nothing is known.

Even this "positive evidence" is a matter of dispute, the cause of which has recently been pointed out by Paul Bert. In many of his ex. periments he failed to demonstrate the contractility of the lungs, but at last he succeeded in doing so; and he found, as the reason of his fail. ure, that he "insufflated the lungs too much", which circumstance impedes their contraction. (Lecons sur la Physiologie comparée de la Re. spiration. Paris, 1870 , p. 375.) But if Paul Bert has thus proven the contractility of the bronchial muscles, he has at the same time conclusively shown the impossibility that asthma can be due to a spasmodic stricture of the bronchial tubes in those cases in which it occurs most frequently, and in which it forms an integral part of the disease, namely, in emphysema. For the same cause which hinders the success of the physiological experiments fully exists under those pathological conditions.

I will not enter into the vast domain of the nervous physiology to rebut step by step the erroneous conclusions which are brought forward as proofs of the nervous origin of asthma. It is too well known that the nervous system easily lends its aid to explain almost the impossible, and why should it fail to do so with regard to asthma? I may the more readily dispense with a similar argumentation, because I am prepared to show that, were even the entire nervous system bent upon producing a spasmodic contraction of the bronchial muscles, it would fail in its attempts, because they are in cases of asthma in such a state of nutrition as to be utterly incapable of contracting at all.

In the vast majority of instances, asthma occurs in connection with

* Abstract of a paper read before the Medlcal Society of London, Nov. 3rd, 1873. 
chronic bronchitis and with emphysema ; $*$ and the rare cases in which these are supposed to be absent could only constitute an exception to the rule. This exception, however, does by no means invalidate the rule. Moreover, there is a certain class of bronchial affections in which cough and expectoration are so slightly marked, that Laennec has described it under the name of latent catarrh. Now, in the acute catarrh, the serous exudation characteristic of this disease remains by no means limited to the mucous membrane alone, but also ex tends to the muscular layer, which is immediately subjacent. So weil has this fact been recognised by all clinical observers, that they more or less explicitly mention "an acute relaxation" (Walshe, Diseases of the Lungs, 1871, page 209) of the bronchial walls in cases of acute bronchitis. Therefore, what holds good in the acute, still more forcibly applies to the chronic affection. Here, indeed, are the bronchial muscles so completely soaked with serum, that, were this the sole change they undergo, it alone would suffice to render contraction of the muscles a matter of impossibility. But very often the first outbreak of an asthmatic attack is preceded by pathological changes of greater moment than bronchitis-namely, by catarrhal pneumonia, the anatomical basis of whooping-cough, measles, etc.; and that disease inflicts such injuries upon the bronchial walls, that their contractility is irrevocably lost.

Gentlemen, we shall never arrive at the true nature of bronchial asthma if we continue to confine our attention to an attack itself, to its immediate causes, and to its peculiar type of respiration. In asthma, as well as in any other disease, the history of the case and its sequelæ will have to be carefully considered; and then we shall find that, indeed, asthma is no independent disease, no dynamic affection of a nerve, but that it really forms a link in a chain of diseases, which commence with affections of the bronchi and terminate with emphysema, whilst asthma itself represents the stage intermediate between these two.

We know of the existence of substantive emphysema, when the thorax is greatly distended, when the loud and sonorous percussion note strangely contrasts with the feeble respiratory murmur, etc. But then the emphysema is formed, and we have to consider that its formation is by no means the pathological work of a day or a week. In what time an atrophic or hypertrophic emphysema developes is not quite known, but we can calculate it approximately, if we consider the time which elapses between the development of a chronic catarrh and that of emphysema; and also the fact that true, substantive emphysema is seldom or never found in children, (Vogel, Lehrbuch der Kinderkrankheiten. Erlangen, 1867, p. 233). From this we must conclude that this destructive work is carried on completely unnoticed by us, and that nothing reveals its existence until the work is finished.

But such profound'changes in the lungs, although they gradually only modify their physical constitution, cannot remain indifferent to their functional activity. As long as no more work is required but that of quiet respiration, this can be performed without great effort on the part of the morbidly altered lung, But when an obstacle arises, which for its removal requires a greater force than that of ordinary respiration, then the inability to effect the removal shows the deficient nutrition of the organ. It is this stage of developing emphysema-this stage of deficient elasticity of the lungs - which constitutes the anatomical basis of bronchial asthma.

The existence of the deficient elasticity of the lungs in asthma does not only follow with necessity from the history of every case, but it is also directly proved by physical signs more delicate than those of auscultation and percussion. Manometric measurements, introduced as a method of diagnosis by Waldenburg ("Die Manometrie der Lungen oder Pneumatometrie als diagnostiche Methode", Berlin. Klin. Wo chensch., No. 45 ; 1871), have shown that emphysema is characterised by insufficient expiration. In individuals suffering from asthma, but who in their free intervals are in every respect considered to be in perfect health, and in whom above all the most careful physical diagnosis could not detect a trace of emphysema, the manometer showed a remarkably insufficient respiratory force, though in less degree than in

* Of the 223 cases tabulated by Dr. Hyde Salter, the original causes of asthma are stated as follows.

Chronic catarrh, cold, and exposure to wet

Whooping-cough, measles, $\ddot{\text { typhoid }}$ fever (cat

Unknown

Smallpox driven in on the lungs $\left(\right.$ sic $^{\circ} \ddot{)}$

Apparently spontaneous

Outbreak and disappearance of

cczema

Liver complaint (!)

Bilious fever (!)

Weak stomach (!) those suffering from emphysema ; and this must be attributed to the same conditions which produce that phenomenon also in emphysema.

The causes and the type of respiration during an asthmatic attack become easily intelligible by the anatomical substratum of asthma which has just been established. In typical bronchial asthma, the lungs are greatly distended, the thorax is in a position of extreme inspiration, and all the respiratory muscles are firmly contracted. Inspiration is short and abrupt, and the effort of the respiratory muscles, although prolonged and greatly increased, remains ineffectual. If an obstacle arise to the entrance and exit of air from the lungs, say the impaction of a tough pellet of mucus in a bronchus, a supplementary force is necessary in order to displace the obstacle and to effectually carry on the respiratory function. Such additional aid to expiration is obtained by deep inspiration; and this is easily effected, because it proceeds from the action of the inspiratory muscles, to which the lungs deficient in elasticity cannot offer any great resistance. Hence the easy and rapid overdistension of the lungs. But this at first diminishes their elasticity, and, only after a deal of the expanding force has been got rid of, the elasticity increases (A. Horvarth, "Zür Lehre von der Elasticität.," Centralblatt, $f . d$. Med. Wissensch., No. 48, 1873), whilst, according to the laws of the self-regulation of respiration through the pneumogastric, the expiratory act is continued until the obstacle is overcome. (J. Breuer, Die Selbststruerung der Athmung durch den Nervus vagus. Sitzungsbericht. der $K . K$. Akad. der Wissensch., Bd. 58, Abth. ii, 1868, p. 909 , et seq.)

The exciting causes of an asthmatic attack either lie in the nature of the disease itself, or external circumstances, which do not affect healthy individuals, but may acquire that importance and produce an attack in the asthmatic. I shall limit myself to a few observations on this subject. The causes are :-

I. A lung deficient in elasticity must, for obvious reasons, more than any other allow of a distension of the blood-vessels, and thus become the fertile soil of hyperæmia and of catarrh. According to the intensity of the irritation, to the nutritive and formative peculiarities of the individual, to the state of the affected blood-vessels, and to that also of the secreting organs, the pathological products of catarrh will be either of a serous, mucous, or more fibrinous (croupous) nature. The removal of these pathological products from the air-passages is essential to the performance of respiration; but the force required to effect it will have to be increased in direct proportion to the tenacity of the sputum and to its more or less firm adhesion to the soil whence it sprung. We have, therefore, to acknowledge the tough and fibrinous sputa as the first cause of an asthmatic attack.

2. The inhalation of foreign bodies, such as ipecacuanha, Asthmatiss ciliaris, Salisbury pollen, etc., and their peculiar action (Blackley, Experimental Researches on the Causes and Nature of Catarrhis Aistivus. London, 1873).

3. The radiation of heat from the surface of the lungs, and the changes which this, on account of its morbid alteration, undergoes in contact with the atmospheric air. The skin of the body is protected by clothes interposed between it and the ambient medium, so that they regulate the radiation of heat from the external surface. The surface of the lung, equally in contact with the atmosphere, possesses, under normal conditions, its protective covering in the water-vapour with which the stagnant atmosphere in the lungs is saturated. But if the blood-vessels exhaling the water-vapour be morbidly changed, if thick mucus cover them like a layer of varnish, then the normal radiation of heat from the pulmonary surfaces is necessarily disturbed; the mucus itself enters into a process of diffusion with the atmosphere, with results varying according to the saturation, temperature, and movement of the latter. Under favourable conditions, the mucus dries up on account of the loss of its watery constituents; and in the lungs the same process takes place, as may be often observed on the mucous membrane of the nose in cases of chronic catarrh, in the eyelids in chronic blepharitis, or if a sore be exposed to the air. In all these cases, crusts form, the nasal-passages are rendered impervious, and the eyelids are sealed together. Similar changes also occur in the lungs. They then require unspeakable efforts on the part of the expiratory forces to get rid of this obstruction.

4. Displacement of secretion accumulated in the lungs from the seat of its formation into another portion of the lungs, in consequence of which the surface of respiration is suddenly greatly diminished (as after laughter). (Beau).

5. Interstitial oedema. It occurs particularly in connexion with diseases of the kidneys (gouty asthma). (Asthma urinosum, Bamberger.)

6. Thrombosis and embolism of the smaller branches of the pulmonary artery. They produce dyspnœea, which cannot during life be referred to its true cause. Its symptoms are those usually ascribed to asthma. The frequency of such occurrence has been pointed out by Virchow, 
and he showed that such pathological conditions existed in from 8 to Io per cent. of the bodies which formed the subject of his anatomical observations. (Lebert, Virchow's Handbuch, 5 Bd., ii Abth. and Lief, I867, pag. 486 .)

All these conditions produce an obstacle to the interchange of gases, against which the deficient expiratory forces will for a long time labour in vain.

I may, therefore, in conclusion, state that asthma is a symptom which accompanies diseases of the lungs in which deficient elasticity is the prominent feature, and in consequence of which the existing expiratory forces are only able to overcome an obstacle to respiration after prolonged and increased efforts. This view of the nature of asthma is, I am confident, in accordance with the anatomical condition of the lungs; and its adoption may not be without practical utility. Whilst hitherto the treatment of asthma mainly consists in the blunting of the nervous system, which, in point of fact, is set to watch over the respiratory organ and to warn it against its dangerous foes, we may, by strict attention to the anatomical basis of asthma, delay for a long time the development of the last link of the pathological chain, as traced above.

\section{ON THE USE OF DISINFECTANTS.}

\section{By E. J. ADAMS, M.R.C.S., L.S.A., L.M., L.R.C.P.E., Medical Officer and Vaccinator.}

AN opinion seems to have sprung up that disinfectants, to be of any practical value, should be used in the concentrated form for the prevention of the spread of disease ; and that heat used for such a purpose should be the red heat; that, in the form they are now employed, the mischief is likely to be increased, instead of arrested. Such a theory would appear to me to be fraught with considerable danger, as the means used in the manner suggested would become a fresh source of risk, and therefore probably discarded altogether, the poison thus being allowed to run riot and gain fresh strength by meeting with kindred spirits of its own.

It being not always possible to capture the enemy alive, we may sometimes succeed in killing and then disposing of him ; therefore, in the case of a room being vacated by a patient after recovery from some communicable illness, the means employed for the purpose, when practicable, should be in a concentrated form; and this gives rise to a suggestion for the universal establishment of Convalescent Homes for the poor, whilst their wretched hovels are undergoing a thorough process of purification and cleansing. To do which effectually, all outlets should be closed and the air thoroughly saturated with chlorine gas or sulphurous acid fumes, the clothes of the patient being allowed to remain in the room during the process; after some hours, the windows may be opened to give place to the outer pure atmosphere. The area of contagion being of limited extent, this proceeding is simply carrying out the precept of "first catching the hare, and then cooking it "; in other words, before it can become distributed by movements in the atmosphere, or by other mechanical means, as by the clothing of people ; and, as an instance of the part it plays in the distribution of disease, a case may be mentioned in which the clothes of two children that had died from fever were put away for seven years, and, then being worn by two other healthy children, they caught the fever and died; the clothes were then destroyed.

We have doubtless, most of us, had practical illustrations of the manner in which the odour of a post mortem may cling to one's attire for many days; therefore, especial attention should be attached to this particular, and all bedding, clothes, and materials used by the patient should be subjected to a temperature not less than 200 deg. Fahr. for several hours, with the addition, perhaps, of sulphur fumes.

Another important matter with reference to the subject is to ensure a plentiful supply of water, the infected house being thoroughly scoured with soap, soda, and water, and the floors sprinkled with disinfecting powder, as it will also cleanse and whiten them; the ceilings re-whitewashed, and a good fire kept burning, which not only helps to purify the air, but also, by creating a current, serves as an excellent means of ventilation, by drawing the air of the room up the chimney, and thus giving place to fresh air from without. Vessels, containing a weak solution of Condy's fluid, may also be placed about the room. For the sick chamber of a well-to-do patient, I would suggest the employment of reversible hydraulic fountains, to be procured at most glass factories, by which means a jet of water coloured with Condy's fluid could be kept playing with a decidedly pretty and beneficial effect. The cost of the apparatus would be simply $£ 2$ or $£ 3$.

As the result of upwards of six years experience of our infirmary, with a yearly average of $\mathbf{I} 20$ midwifery cases, together with numerous cases of phlegmonous erysipelas, variola, scarlatina, choleraic diarrhoea, with an occasional case or two of puerperal fever from without, I cannot recollect a single case of communication of disease through infection, and should not hesitate to consider a room perfectly fit for habitation after having been occupied by an infectious case, provided proper care had been taken to use the remedies recommended. And, in a case of scarlet fever unconsciously occurring in a family during a wedding, whilst several friends were sleeping at the house, besides a large family of children, some of whom had not had the complaint, not one fresh case occurred, and the room was shortly after occupied by other children.

All the excreta of patients should be freely mixed with moderately strong disinfectants as soon as possible, and the so-called disinfecting powder, consisting of a mixture of carbolate of lime and hyposulphite or hypochlorite of lime, might be advantageously made use of. The hypochlorite, when used too liberally, often produces great tingling and smarting of the eyes and eyelids, and the smell of it induces nausea. The nitrate of benzole is thought to be effectual in removing this annoyance. The patient might even be sponged, where practicable, with tepid water containing a little Condy's fluid, or even the hyposulphite of soda or potash, which will prove very grateful; and the hyposulphites might even be given internally, combined with cinchona and port wine ; myrrh, as an antiseptic, might, perhaps, be added. All bad smells should be traced to their origin, and the cause, if possible, removed. It is an important fact that a foul atmosphere predisposes to puerperal fever. It may be taken as a rule that bad air induces fever, forming, as it were, a hotbed for it ; and disinfectants, by destroying chemically the products of decomposition, must also exercise a destructive influence upon fever-poison, whether of vegetable or animal origin, and these are its most fertile causes; and, the more complex the matter, the greater the number and variety of changes, and hence the more noxious the result.

Although, perhaps, not directly generating zymotic disease, bad gases produce a set of symptoms analogous in character, and at least prevent the pure air-nature's purifier-from coming into actual contact with the decomposing matter. The poisonous effect of gases is observed in their depressing influence on the system, thus rendering the body more prone to fever, and less able to resist its influence. The different species of germs (monads and others) exist mostly in the lower strata of inhabited towns, and in the Alps are almost absent.

It has been said, that small cells resembling pus-cells exist in the air of a ward containing cases of epidemic ophthalmia, or suppurating surfaces of any kind, and, multiplying, convert the tissues by which they are surrounded into structures like unto themselves, as in variola, etc. Experiments have been made upon mity cheese by subjecting it to the action of chlorine gas, with varying results; and meat, when boiled with chlorine, becomes completely broken up. Carbolic acid, when strong enough to destroy venous, acetous, and lactic fermentation, does not arrest the conversion of starch into sugar by diastase, nor the formation of oil of bitter almonds, having no action upon substances of purely chemical composition, though putting a stop to changes due to organic life. It also destroys the vitality of vaccine lymph. Sesquichloride of iron decomposes sulphuretted hydrogen and the ammonia sulphide, though carbolic acid does not. Sulphurous acid acts in a similar manner to chlorine, and is especially destructive to animal life, as evinced by its being employed as a specific in psora and other diseases of parasitic origin.

It has been argued that, during the Franco-German war, the air of the hospital, notwithstanding the use of carbolic acid, was most impure ; but the fact of excessive overcrowding is lost sight of, and we are not told what local means were employed as a preventive. We might have hoped that surgery, as a science, had advanced so far that suppuration had become a matter of rarity after surgical operations, and it would be interesting to know how far the carbolic or chloride of zinc treatment had been carried out. Moreover, at Jarrow, although the air is pervaded by chlorine gas from the various factories, fever and small-pox are said to abound ; yet we hear nothing about the drainage of the town, nor to what extent vaccination is practised.

We should endeavour, if possible, to get at the cause, not simply the effect, otherwise one might as well hope to get rid of weeds by cutting the stalks off without destroying the roots. At the Hampstead Hospital, the precautionary measures are so complete that not a case is known where variola has been communicated by the patient or his clothes after leaving, although clad in the same attire as when admitted; and at Terling there are good grounds for believing that the free use of disinfectants in their present form cut short its ravages.

Heat, as an agent for disinfection, should be dry; a moist heat seems to favour noxious exhalations. It would be as well for medical men to pay great attention to their hands and nails at all times, keeping the 